\title{
Chaos Control and Stabilization of a PID Controlled Buck Converter Using the Spotted Hyena Optimizer
}

\author{
Derradji Bakria \\ LAADI Laboratory \\ Department of Sciences and Technology \\ Ziane Achour University of Djelfa \\ Djelfa, Algeria \\ bakriaderradji@gmail.com
}

\author{
Messaouda Azzouzi \\ Department of Sciences and Technology \\ Ziane Achour University of Djelfa \\ Djelfa, Algeria \\ Dr.Azzouzi@yahoo.fr
}

\author{
Djamal Gozim \\ Department of Sciences and Technology \\ Ziane Achour University of Djelfa \\ Djelfa, Algeria \\ gozimansour@gmail.com
}

\begin{abstract}
The voltage controlled buck converter by constantfrequency pulse-width modulation in continuous conduction mode gives rise to a variety of nonlinear behaviors depending on the circuit parameters values, which complicate their analysis and control. In this paper, a description of the DC/DC buck converter and an overview of some of its chaotic dynamics is presented. A solution based on the optimized PID controller is suggested to eliminate the observed nonlinear phenomena and to enhance the dynamics of the converter. The parameters of the controller are optimized with the Spotted Hyena Optimizer (SHO) which uses the sum of the error between the reference voltage and the output voltage as well as the error between the values of the inductor current in every switch opening instant to determine the fitness of each solution. The simulations results in MATLAB proved the efficiency of the proposed solution.
\end{abstract}

Keywords-buck converter; nonlinear behavior; optimized PID controller; spotted hyena optimizer

\section{INTRODUCTION}

DC-DC switched mode power converters have been widely used in many industrial and domestic applications such as hybrid cars, photovoltaic solar energy applications, cell phones chargers, etc. due to their high efficiency compared to the conventional linear regulators. To achieve the desired performance, the DC-DC converters can be either current controlled or voltage controlled. Controllers based on linear theory can be used due to their easy and low implementation cost and the good performance around the operating point [1, 2]. However, they do not take into consideration the nonlinear phenomena that can occur in the converter in high frequencies or when one of the parameters of the converter changes [3-6]. Recent research has proposed numerous solutions to address the above-mentioned problems. For instance, in [7], boost converter discrete-time models were used as an example to present an analytical solution that allows the prediction of nonlinear phenomena in digital current mode controlled power converters. Meanwhile, in [8] the authors proposed a new method for the design of a stable and robust feedback control using the bifurcation analysis of the bilinear averaged model of boost converter and constrained stabilization principles. Another approach for designing a controller that guarantees the system's global stability was introduced in [9]. The approach is based on the principles of the contraction theory of switched systems. Nonetheless, the aforementioned works are based on analytical solutions and are a bit complex, and hard to implement in real plants. Fuzzy logic-based controllers have been used to control chaos in many studies [10-18]. However, the use of fuzzy logic complicates the control system and requires considerable computation time and a large memory space, while the use of the controllers based on metaheuristic algorithms ensure, at least, the same performance as the fuzzy controllers with less complexity and implementation cost [1921]. Nonetheless, most of previous works that use this type of controller are using the Integral Absolute Error (IAE) as an adaptation coefficient which is insufficient to estimate the fitness of each solution during the search.

Nonlinear phenomena elimination in power converters is still an open research subject, although good results have been achieved in the literature. In this paper, we propose a method of shifting the nonlinear phenomena and expand the stability range of the converters without the need of complicated analysis methods or additional circuits. Using the powerful tools of MATLAB and the state-space model of the buck converter as an example, we designed a PID regulator that guarantees both global stability of the system and parameter disturbance rejection (load, input voltage, reference voltage). This design is based on the Spotted Hyena Optimizer (SHO) to determine the optimum gains of the controller using appropriate adaptation coefficients. 


\section{SYSTEM DESCRIPTION}

\section{A. Buck Converter}

A buck converter is a Switch Mode Power Supply (SMPS) that converts a DC voltage into a lower DC voltage through the commutation between different configurations [22, 23]. Buck converters are generally controlled via voltage mode control. In this control mode, the error $(\mathcal{E}(t))$ between the output voltage $\left(V_{o}(t)\right)$ and the reference voltage $\left(V_{\text {ref }}\right)$ is used to obtain the control signal $(\mathrm{Cm}(t))$. The latter is compared with the sawtooth signal to generate the PWM signal needed to derive the switch. The proposed study circuit is illustrated in Figure 1.

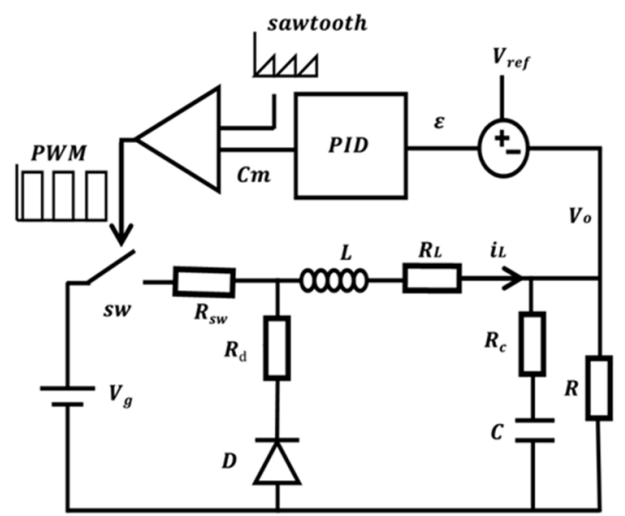

Fig. 1. Voltage controlled buck converter.

The state of the system is expressed by the vector $X=\left[i_{L}, V_{C}\right]^{T}$ with:

$$
\dot{\mathrm{X}}_{l}=A_{i} \mathrm{X}_{i}+B_{i}
$$

where the state matrices $A_{i}$ and $B_{i}$ for each configuration are:

$$
\begin{gathered}
A_{1}=\left[\begin{array}{cc}
-\frac{\left(R_{S w}+R_{L}+\left(\frac{R_{C} R}{R_{C}+R}\right)\right)}{L} & \frac{-R}{L\left(R+R_{C}\right)} \\
\frac{R}{C\left(R+R_{C}\right)} & \frac{-1}{C\left(R+R_{C}\right)}
\end{array}\right], B_{1}=\left[\begin{array}{l}
\frac{V_{g}}{L} \\
0
\end{array}\right] \\
A_{2}=\left[\begin{array}{cc}
-\frac{\left(R_{D}+R_{L}+\left(\frac{R_{C} R}{R_{C}+R}\right)\right)}{L} & \frac{-R}{L\left(R+R_{C}\right)} \\
\frac{L}{C\left(R+R_{C}\right)} & \frac{-1}{C\left(R+R_{C}\right)}
\end{array}\right], B_{2}=\left[\begin{array}{l}
0 \\
0
\end{array}\right] \\
A_{3}=\left[\begin{array}{cc}
0 & 0 \\
0 & \frac{-1}{C\left(R+R_{C}\right)}
\end{array}\right], B_{3}=\left[\begin{array}{l}
0 \\
0
\end{array}\right]
\end{gathered}
$$

Equation (5) represents the solution of (1):

$$
\mathrm{X}_{i}(t)=e^{A_{i}\left(t-t_{0}\right)}\left(\mathrm{X}_{i}\left(t_{0}\right)+A_{i}^{-1} B_{i}\right)-A_{i}^{-1} B_{i}
$$

The behavior of the system is described in each configuration using (5) where the final state of the system in the current configuration is considered as the initial state $\mathrm{X}_{i}\left(t_{0}\right)$ for the next one.

\section{B. Spotted Hyena Optimizer}

SHO imitates the natural hunting behavior of the spotted hyenas to find the optimum solution for an optimization problem $[24,25]$. A pack of spotted hyenas follow an organized group hunting technique that can be divided into three main behaviors:

\section{1) Searching and Encircling Prey}

When the hunt begins, the pack of the spotted hyenas has no information about the position of the prey, therefore the current fittest search agent will decide the possible location of the prey in the search space. Then, the rest of the pack will surround this position.

\section{2) Hunting}

The group hunting behavior of the spotted hyenas is based on a cluster of trusted friends that guide the members of the pack during the hunt.

\section{3) Attacking}

All search agents will be forced to move towards the position of the prey.

More details on the mathematical model of the SHO algorithm can be found in [24, 25].

\section{SHO Application}

In this study, the SHO algorithm was applied to determine the optimal parameters of a PID controller that would improve the dynamics of the converter. It is based on the minimization of the steady state error between the reference and the measured voltage, and the error between the successive values of the inductor current (measured in each switch opening instant) which are the peaks of the inductor current. Equation (6) represents the proposed objective function.

$$
\begin{gathered}
F(t)=w_{1} F_{1}+w_{2} F_{2} \\
F_{1}=\sqrt{\frac{\sum_{i}^{N}\left(P_{i}-\mu\right)^{2}}{N}} \\
F_{2}=\int_{t_{0}}^{t_{f}}|\varepsilon(t)| d t
\end{gathered}
$$

where $F_{1}$ is the standard deviation of inductor current values measured in each switch opening instant, and $F_{2}$ represents the Integral Absolute Error (IAE).

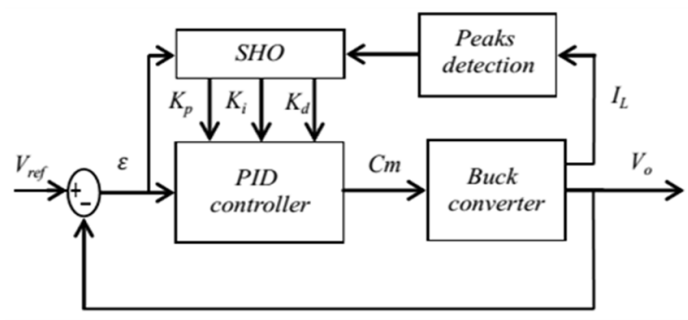

Fig. 2. Closed loop system with SHO

Figure 2 presents the control block diagram of the system, where $K_{p}, K_{i}$ and $K_{d}$ are respectively the proportional, the integral, and the derivative gains obtained by the SHO.

\section{RESULTS AND DISCUSSION}

The mathematical model of the proposed circuit presented in Figure 1 was simulated in MATLAB using (2)-(5). The converter and controller parameters are presented in Table I. 
TABLE I. PARAMETERS OF THE PROPOSED CIRCUIT

\begin{tabular}{|c|c|c|}
\hline \multirow{4}{*}{ Converter } & $V_{g}$ & $24 \Omega$ \\
\cline { 2 - 3 } & $F$ & $2500 \mathrm{~Hz}$ \\
\cline { 2 - 3 } & $R_{s w}=R_{D}$ & $0.0177 \mathrm{~V}$ \\
\cline { 2 - 3 } & $L$ & $0.02 \mathrm{H}$ \\
\cline { 2 - 3 } & $R_{L}$ & $2 \Omega$ \\
\cline { 2 - 3 } & $C$ & $47 \mu F$ \\
\cline { 2 - 3 } & $R_{C}$ & $0.2 \Omega$ \\
\hline \multirow{4}{*}{ Optimizer } & $R$ & $22 \Omega$ \\
\cline { 2 - 3 } & $w_{1}$ & 100 \\
\cline { 2 - 3 } & $w_{2}$ & 1 \\
\cline { 2 - 3 } & Iterations & 100 \\
\hline \multirow{4}{*}{ Cearch agents } & 20 \\
\hline & $K_{p}$ & 8 \\
\cline { 2 - 3 } & $K_{i}$ & 800 \\
\cline { 2 - 3 } & $K_{d}$ & 0.0001 \\
\hline
\end{tabular}

\section{A. Original Behavior}

In this study, the input voltage was chosen for study and observation while the other parameters had fixed values. As shown in Figure 3, the converter dynamics clearly changed from Period 1, to Period 2, to Period 4, and then to chaotic dynamic behavior.

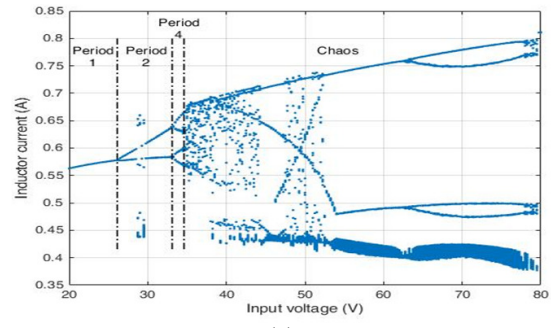

(a)

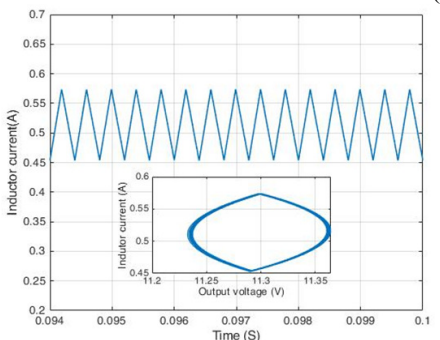

(b)

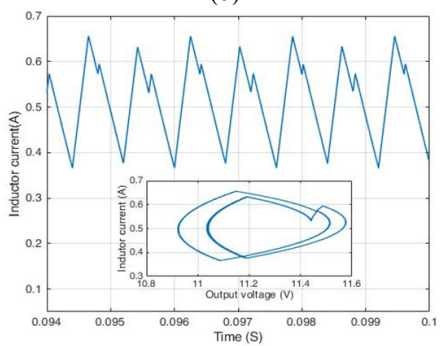

(d)

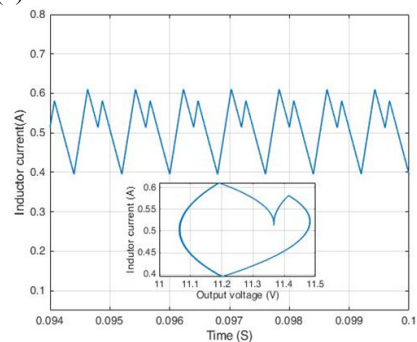

(c)

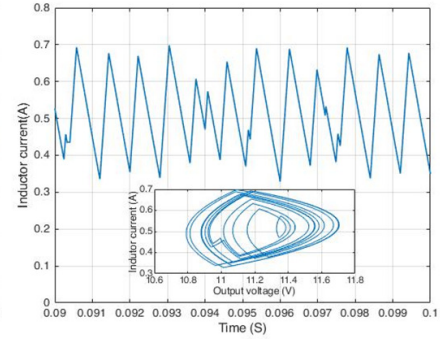

(e)
Fig. 3. (a) Bifurcation diagram varying $V_{g}$. (b) Phase plan diagram $V_{o}-i_{L}$ and the inductor current $i_{L}(t)$ of Period 1 when $V_{g}=24 V$. (c) Phase plan diagram $V_{o}-i_{L}$ and the inductor current $i_{L}(t)$ of Period 2 when $V_{g}=30 \mathrm{~V}$. (d) Phase plan diagram $V_{o}-i_{L}$ and the inductor current $i_{L}(t)$ of Period 4 when $V_{g}=34 V$. (e) Phase plan diagram $V_{o}-i_{L}$ and the inductor current $i_{L}(t)$ of chaos when $V_{g}=40 \mathrm{~V}$.
The input voltage $V_{g}$ was set to start from $20 \mathrm{~V}$, and then it increased gradually to $80 \mathrm{~V}$. The converter exhibits the stable Period 1 behavior when $V_{g} \leq 26 \mathrm{~V}$ followed by the unstable period 2 behavior when $26<V_{g} \leq 33.25 V$. A fterwards, the converter exhibits the unstable Period 4 when $33.25<V_{g} \leq$ $34.75 \mathrm{~V}$. Finally, the converter shows irregular behavior (chaos) when $V_{g}>34.75 V$. Figures 3(b), 3(c), 3(d) and 3(e) show the phase plan diagram $V_{o}-i_{L}$ and the inductor current $i_{L}(t)$ of the aforementioned behaviors respectively.

\section{B. Optimized Behavior}

As shown in the convergence curve in Figure 4, the SHO algorithm finds the optimal gains of the controller after a few iterations. Figure 5 is the voltage wave diagram obtained before and after the optimized PID controller was implemented, where the latter begins after $0.06 \mathrm{~s}$. It is clear from the wave form of the output voltage and the phase plan diagram $V_{o}-i_{L}$ that the converter returns to the stable Period 1 behavior at $0.067 \mathrm{~s}$.

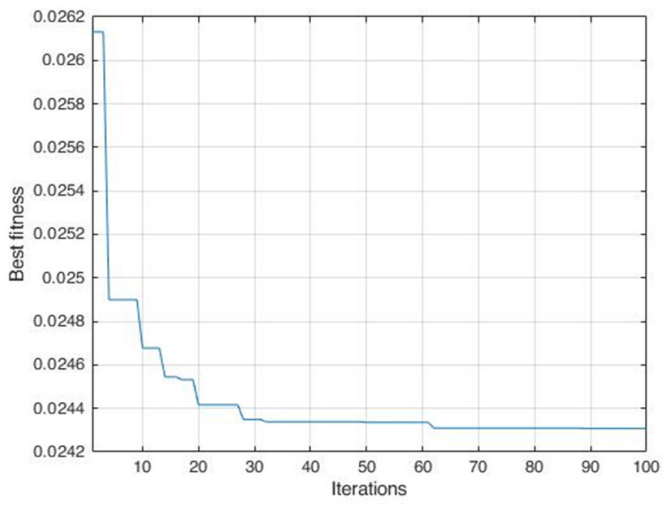

Fig. 4. Convergence curve.

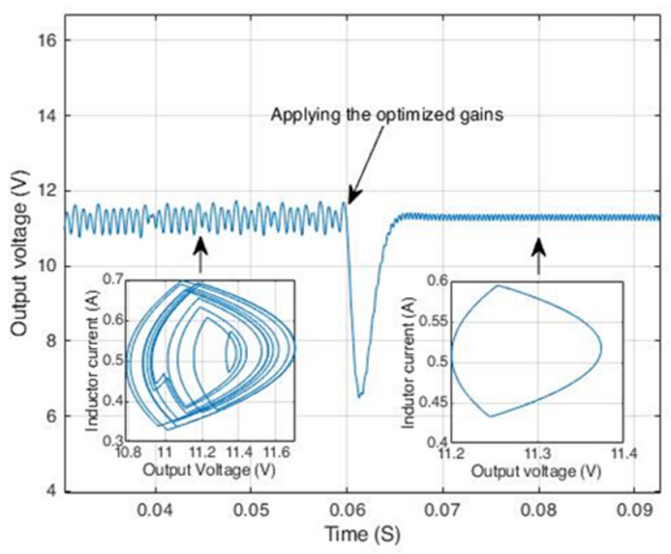

Fig. 5. $\mathrm{V}_{o}$ after applying the optimized parameters.

The bifurcation diagram in Figure 6 proves that the optimized controller is able to extend the stability range of the converter up to $80 \mathrm{~V}$ of $V_{g}$. For more efficiency evaluation, the robustness of the control to the sudden changes in the parameters of the converter has been tested. The response of the output voltage $V_{o}$ to a sudden change in the input voltage, the reference voltage and load are presented in Figure 7. 


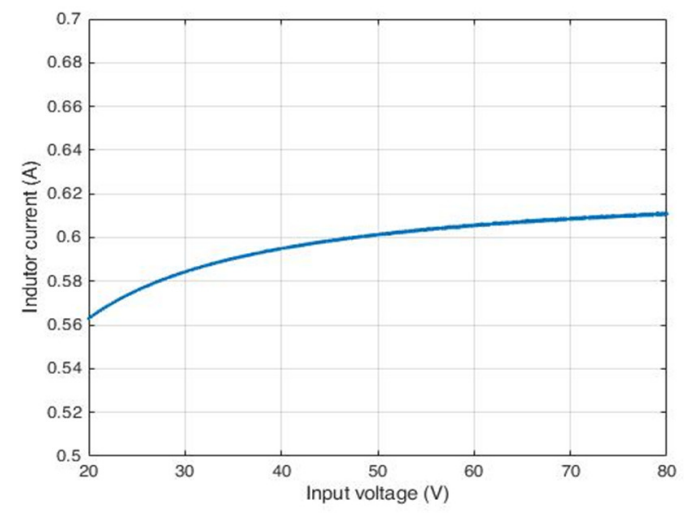

Fig. 6. Bifurcation diagram varying $V_{g}$ using the optimized PID.

(a)

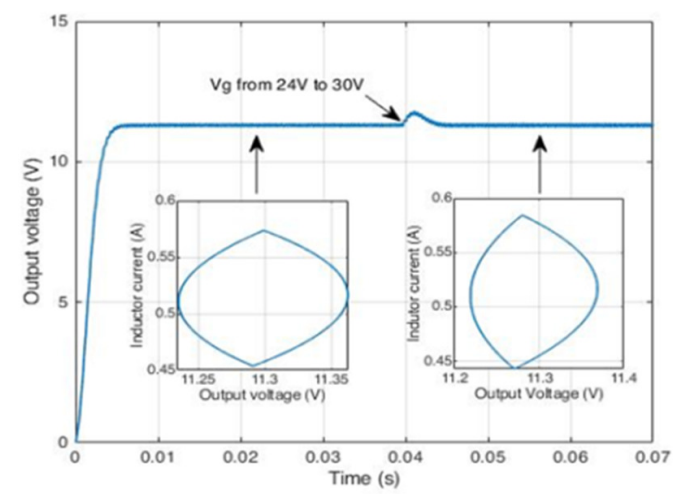

(b)

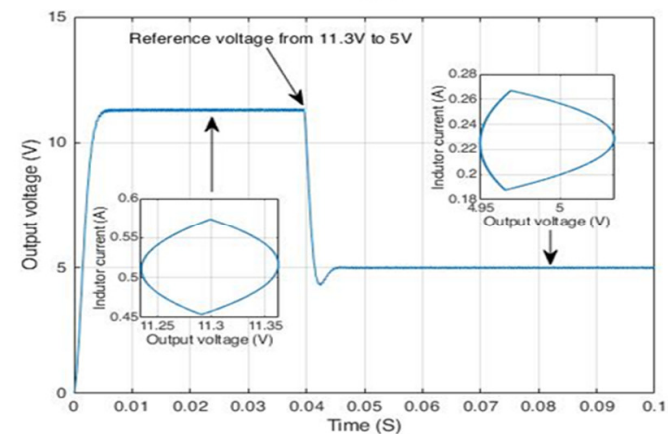

(c)

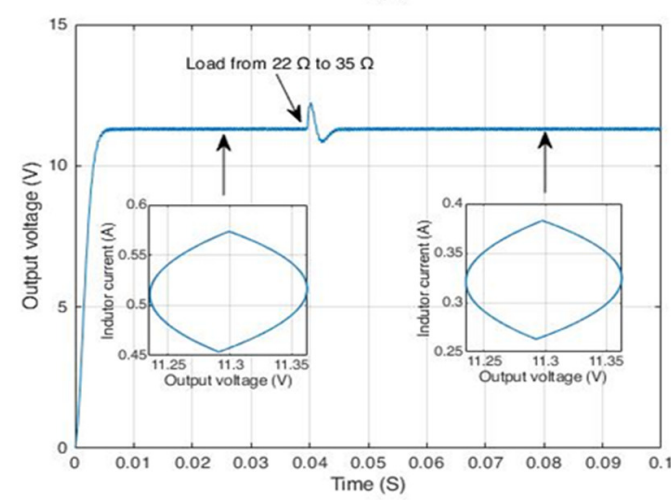

Fig. 7. System response to changes in: (a) Input voltage, (b) reference voltage, (c) load.

In the first $40 \mathrm{~ms}$, the system is subject to the parameters given in Table I, as shown in Figure 7(a). Steady state is reached in $6 \mathrm{~ms}$ with no overshoot. At $t=40 \mathrm{~ms}$, the input voltage is increased to $30 \mathrm{~V}$, as it is remarked, and the controller was able to stabilize the system. At the same instant as before, we repeated the same scenario by inducing a sudden change in the reference voltage. At $\mathrm{t}=40 \mathrm{~ms}$, the reference voltage changed to $5 \mathrm{~V}$, and the controller adapts with the change and stabilizes the system to the new operating point. The results are shown in Figure $7(\mathrm{~b})$. The same scenario is repeated again to test the response of the system to the change in load. At $\mathrm{t}=40 \mathrm{~ms}$ we changed the load to $35 \Omega$. From Figure 7(c), it can be seen that the system remains stable and tracks the reference voltage.

\section{CONCLUSION}

In this paper, PI D controller gain tuning for a voltage controlled DC-DC buck converter is formulated as an optimization problem. This optimization is aimed to produce gains for the controller that would guarantee a robust closed loop. In addition, the objective function used to evaluate the fitness of the gains during the optimization is based on the sum of the error between the reference voltage and the output voltage as well as the error between the values of the inductor current in every switch opining instant. The SHO was used to solve this problem.

To evaluate the efficiency of the designed controller to shift nonlinear phenomena, the bifurcation diagram obtained using the optimized controller is compared to that presented in the literature. We also tested the capability of the controller to reject the disturbance in the parameters of the converter (load, input voltage, and reference voltage). The obtained results prove the efficiency of the proposed solution to shift the nonlinear phenomena and to expand the range of the desired period 1 behavior. In addition to its high efficiency, this solution offers a simple control circuit and low implementation cost without the need of additional circuits.

\section{REFERENCES}

[1] A. G. Perry, G. Feng, Y.-F. Liu, and P. C. Sen, "A Design Method for PI-like Fuzzy Logic Controllers for DC-DC Converter," IEEE Transactions on Industrial Electronics, vol. 54, no. 5, pp. 2688-2696, Oct. 2007, https://doi.org/10.1109/TIE.2007.899858.

[2] M. Saoudi, A. El-Sayed, and H. Metwally, "Design and implementation of closed-loop control system for buck converter using different techniques," IEEE Aerospace and Electronic Systems Magazine, vol. 32, no. 3, pp. 30-39, Mar. 2017, https://doi.org/10.1109/MAES.2017. 150261 .

[3] M. M. Al-Hindawi, A. Abusorrah, Y. Al-Turki, D. Giaouris, K. Mandal, and S. Banerjee, "Nonlinear Dynamics and Bifurcation Analysis of a Boost Converter for Battery Charging in Photovoltaic Applications," International Journal of Bifurcation and Chaos, vol. 24, no. 11, Nov. 2014, Art. no. 1450142, https://doi.org/10.1142/S0218127414501429.

[4] A. Ghosh and S. Banerjee, "Study of complex dynamics of DC-DC buck converter," International Journal of Power Electronics, vol. 8, no. 4, pp. 323-348, Jan. 2017, https://doi.org/10.1504/IJPELEC.2017.085200.

[5] S. Banerjee, A. Ghosh, and S. Padmanaban, "Modeling and analysis of complex dynamics for dSPACE controlled closed-loop DC-DC boost converter," International Transactions on Electrical Energy Systems, vol. 29, no. 4, 2019, Art. no. e2813, https://doi.org/10.1002/etep.2813.

[6] S. Demirbas, H. Fidanboy, and E. Kurt, "Exploration of the Chaotic Behaviour in a Buck-Boost Converter Depending on the Converter and Load Elements," Journal of Electronic Materials, vol. 8, no. 45, pp. 3889-3899, 2016, https://doi.org/10.1007/s11664-016-4450-4. 
[7] A. K. Singha, S. Kapat, S. Banerjee, and J. Pal, "Nonlinear Analysis of Discretization Effects in a Digital Current Mode Controlled Boost Converter," IEEE Journal on Emerging and Selected Topics in Circuits and Systems, vol. 5, no. 3, pp. 336-344, Sep. 2015, https://doi.org/ 10.1109/JETCAS.2015.2462151.

[8] C. Yfoulis, D. Giaouris, F. Stergiopoulos, C. Ziogou, S. Voutetakis, and S. Papadopoulou, "Robust constrained stabilization of boost DC-DC converters through bifurcation analysis," Control Engineering Practice, vol. 35, pp. 67-82, Feb. 2015, https://doi.org/10.1016/j.conengprac. 2014.11.004.

[9] D. Angulo-Garcia, F. Angulo, G. Osorio, and G. Olivar, "Control of a DC-DC Buck Converter through Contraction Techniques," Energies, vol. 11, no. 11, Nov. 2018, Art. no. 3086, https://doi.org/10.3390/ en1 1113086

[10] K. Guesmi, N. Essounbouli, A. Hamzaoui, J. Zaytoon, and N. Manamanni, "Shifting nonlinear phenomena in a DC-DC converter using a fuzzy logic controller," Mathematics and Computers in Simulation, vol. 76, no. 5, pp. 398-409, Jan. 2008, https://doi.org/ 10.1016/j.matcom.2007.04.008.

[11] K. Guesmi, N. Essounbouli, and A. Hamzaoui, "Systematic design approach of fuzzy PID stabilizer for DC-DC converters," Energy Conversion and Management, vol. 49, no. 10, pp. 2880-2889, Oct. 2008, https://doi.org/10.1016/j.enconman.2008.03.012.

[12] K. Mehran, D. Giaouris, and B. Zahawi, "Stability Analysis and Control of Nonlinear Phenomena in Boost Converters Using Model-Based Takagi-Sugeno Fuzzy Approach," IEEE Transactions on Circuits and Systems I: Regular Papers, vol. 57, no. 1, pp. 200-212, Jan. 2010, https://doi.org/10.1109/TCSI.2009.2019389.

[13] W. Hu, B. Zhang, and R. Yang, "Bifurcation Mechanism and Stabilization of V2C Controlled Buck Converter," IEEE Access, vol. 7, pp. 77174-77182, 2019, https://doi.org/10.1109/ACCESS.2019. 2918297.

[14] G. Djamal, G. Kamel, and M. Djiali, "On the elimination of nonlinear phenomena in DC/DC converters using type-2 fuzzy logic controller," Diagnostyka, vol. 19, no. 3, pp. 73-80, Sep. 2018, https://doi.org/ 10.29354/diag/93139.

[15] M. Ayati, A. Bakhtiyari, and A. Gohari, "Chaos control in buck converter using fuzzy delayed-feedback controller," in 4th International Conference on Control, Instrumentation, and Automation, Qazvin, Iran, Jan. 2016, pp. 362-365, https://doi.org/10.1109/ICCIAutom.2016. 7483189.

[16] Y. Yuan, C. Chang, Z. Zhou, X. Huang, and Y. Xu, "Design of a singleinput fuzzy PID controller based on genetic optimization scheme for DC-DC buck converter," in International Symposium on NextGeneration Electronics, Taipei, Taiwan, May 2015, pp. 1-4, https://doi.org/10.1109/ISNE.2015.7131965.

[17] Z. B. Duranay, H. Guldemir, and S. Tuncer, "Fuzzy Sliding Mode Control of DC-DC Boost Converter," Engineering, Technology \& Applied Science Research, vol. 8, no. 3, pp. 3054-3059, Jun. 2018, https://doi.org/10.48084/etasr.2116.

[18] K. Behih, K. Benmahammed, Z. Bouchama, and M. N. Harmas, "RealTime Investigation of an Adaptive Fuzzy Synergetic Controller for a DC-DC Buck Converter," Engineering, Technology \& Applied Science Research, vol. 9, no. 6, pp. 4984-4989, Dec. 2019, https://doi.org/10.48084/etasr.3172.

[19] H. Abderrezek, A. Ameur, and M. N. Harmas, "Robust DC/DC converter controllers using PSO," Electrotehnica, Electronica, Automatica, vol. 65, no. 1, pp. 31-37, Jan. 2017.

[20] C.-B. Fu, A.-H. Tian, K.-N. Yu, Y.-H. Lin, and H.-T. Yau, "Analyses and Control of Chaotic Behavior in DC-DC Converters," Mathematical Problems in Engineering, vol. 2018, Nov. 2018, Art. no. e7439137, https://doi.org/10.1155/2018/7439137.

[21] H. Abdelgawad and V. Sood, "Boost Converter Controller Design Based On Particle Swarm Optimization," International Journal on Power Engineering and Energy, vol. 7, no. 2, pp. 647-659, 2016.

[22] R. W. Erickson and D. Maksimovic, Fundamentals of Power Electronics. Netherlands, Amsterdam: Kluwer Academic Publishers, 2001.
[23] K. Jayaswal and D. K. Palwalia, "Performance Analysis of Non-Isolated DC-DC Buck Converter Using Resonant Approach," Engineering, Technology \& Applied Science Research, vol. 8, no. 5, pp. 3350-3354, Oct. 2018, https://doi.org/10.48084/etasr.2242.

[24] G. Dhiman and V. Kumar, "Spotted hyena optimizer: A novel bioinspired based metaheuristic technique for engineering applications," Advances in Engineering Software, vol. 114, pp. 48-70, Dec. 2017, https://doi.org/10.1016/j.advengsoft.2017.05.014.

[25] G. Dhiman and A. Kaur, "Spotted Hyena Optimizer for Solving Engineering Design Problems," in International Conference on Machine Learning and Data Science, Noida, India, Dec. 2017, pp. 114-119, https://doi.org/10.1109/MLDS.2017.5. 